\title{
Editorial
}

\section{Imminent threat of antibiotic resistance and the importance of diagnostic and antibiotic stewardship}

\author{
Erlangga Yusuf \\ Department of Medical Microbiology, Antwerp University Hospital (UZA), \\ University of Antwerp, Belgium
}

\begin{abstract}
Antibiotics are one of the hallmarks of modern medicine. Antibiotics have saved many lives that was unthinkable prior to their discovery. Yet, antibiotic use is associated with the danger of bacterial resistance. Bacterial resistance to antibiotics means simply that antibiotics cannot be used to treat infection, that a simple skin and soft tissue infection may lead to death. Already in 1945, when Alexander Fleming received his Nobel Prize for his part in the discovery of penicillin, he delivered a speech where he warned about this danger. ${ }^{(1)}$ In his speech, he mentioned the problem of underdosage of antibiotics. As the time passed by, we know that the problem is not only related to underdosage but also to other aspects of inappropriate use of antibiotics such as unnecessary use (for example when the infection is due to a virus), and extended duration of antibiotics. ${ }^{(2)}$

The danger of resistance is imminent. In less than 20 years, extended spectrum beta-lactamase (ESBL), i.e. resistance of gram-negative bacteria to third generation cephalosporines has become common in many medical institutions in the world. ${ }^{(3)}$ Extended spectrum beta-lactamase bacteria can still be treated with antibiotics from the carbapenem class, but resistance to this type of antibiotic has emerged too in the so-called carbapenemase-producing Enterobacteriaceae (CPE). When CPE bacteria are found in a patient, not many other antibiotic options are left and resistance to last option antibiotics has been described. Two years ago, the world was shocked
\end{abstract}

when a group from China reported the finding of the genes that coded for the resistance to colistin. ${ }^{(4)}$ While the speed of the emergence of bacterial resistance is high, the speed of the discoveries of new antibiotics is slow due to economical and scientific reasons. ${ }^{(5)}$ Unlike chemotherapeutic or anti-rheumatoid drugs for example, antibiotics are normally used for short term treatment and they are financially not promising for pharmaceutical industries. Developing of new antibiotics is also hampered by the necessity to find targets in the bacteria that are not prone to rapid development of resistance and by problems with bacterial entry and efflux in Gram-negative organisms. Since the development of new antibiotics is so difficult, we have no choice at this moment except to conserve the antibiotics that we still have. To that end, diagnostic and antibiotic stewardship should be put in place.

Diagnostic stewardship is defined as coordinated guidance and interventions to improve appropriate use of microbiological diagnostics to guide therapeutic decisions. In other words, diagnostic testing is needed to establish the presence or absence of infection. ${ }^{(6)}$ While the term diagnostic stewardship is relatively new, the term is antibiotic stewardship is already in place for a while. The idea of antibiotic stewardship is to have coherent actions which promote using antimicrobials responsibly. ${ }^{(7)}$ The most important similarity of both terms is the 'coordinated action'. To 
achieve the aim, all actors should have a role and work together in concerted action. The prescriber should make a diagnosis of infection based on clinical judgement. He should perform a diagnostic test, either culture or serology based. $\mathrm{He}$ can be aided by the clinical microbiologist who has the knowledge of the performance of a test. The clinical microbiologist has a very important role in preventing misuse of microbiological tests. For example, taking a superficial swab for a skin and soft tissue infection should be discouraged since the finding of skin microorganisms will prompt unnecessary antibiotic therapy. When the clinician decides that an antibiotic is really needed, he should give the antibiotic in the correct dose, right duration and whenever possible by the oral mode of administration. The finding of the pathogen, and the antimicrobial susceptibility should be reported timely by the laboratory, within 48 to 72 hours. Based on the laboratory results the empiric antibiotic that has been started should be de-escalated to a narrower spectrum. When multidrug resistant organisms (MDRO) such as ESBL and CPE are found in a patient, the patient should be isolated to prevent further spread of MDRO. This action should be coordinated by infection prevention nurses and nurses of the ward. The pharmacologist should aid the clinician with the right dose of antibiotics. Since all of this should be coordinated, the role of the hospital management is also important. They should maintain the diagnostic and antibiotic stewardship process. The process should be continuously present and not only as a short program. In some countries in Europe the management is aided by an antimicrobial stewardship team as requested by law.

Clearly, this coordinated effort needs financial resources. In middle-income countries such as Indonesia, the healthcare system with plenty of financial resources is the private one. However, in private healthcare, there is somehow the emphasis on pleasing the patient, so that antibiotics are perhaps prescribed more often than in public healthcare. The private healthcare system has also no financial benefit by containing MDRO spread since the patients infected by MDRO will stay longer in the hospital and ironically enough it will generate more money for the private hospital. In general, as a middle-income country, like many other countries in South East Asia, Indonesia's healthcare system is also not bestowed with plenty of financial resources, and diagnostic and antimicrobial stewardship are also rare. ${ }^{(8)}$

Simply copying the implementation of diagnostic and antimicrobial stewardship from high income countries is surely not possible for a middle-income country. The diagnostic and antimicrobial stewardship in high-income countries are also merely oriented to second and third line healthcare, i.e. hospitals and not the first line care. First line care is perhaps the setting where the most antibiotics are prescribed in middle-income countries. Some adaptation is clearly needed but the most important thing is political support. The ministry of health should put the fight against antimicrobial resistance as priority. Awareness of the danger of antimicrobial resistance should be raised not only for the doctors but also for the patients. Medical faculties and scientific associations for the general practitioners and other medical specializations must be involved. Social media should be used to promote the campaign to teach the patients that antibiotics are not always needed. Regulation for sure is also needed on curbing and stopping over the counter and counterfeit antibiotics. It is also important to perform surveillance of antibiotics use and the prevalence of MDRO. These data can perhaps be obtained by health authorities or statistics offices. These data are important in order to be able to react immediately when an increasing trend is found.

Antibiotic resistance is an imminent problem, the threat is real, and the solution to this problem is not easy. Political will is needed and due to financial limitation and private oriented health care system, diagnostic and antimicrobial stewardship as performed in highincome countries should be adapted locally. 


\section{REFERENCES}

1. Fleming A. Nobel lecture: penicillin. Nobel Lect Physiol or Med 1945;83-93.Available at: https:// www.nobelprize.org/nobel_prizes/medicine/ laureates/1945/fleming-lecture.pdf. Accessed October 12, 2017.

2. Morency-Potvin P, Schwartz DN, Weinstein RA. Antimicrobial stewardship: how the microbiology laboratory can right the ship. Clin Microbiol Rev 2017;30:381-407.

3. Gniadkowski M. Evolution and epidemiology of extended-spectrum $\beta$-lactamases (ESBLs) and ESBL-producing microorganisms. Clin Microbiol Infect 2001;7:597-608.

4. Liu YY, Wang Y, Walsh TR, et al. Emergence of plasmid-mediated colistin resistance mechanism
MCR-1 in animals and human beings in China: a microbiological and molecular biological study. Lancet Infect Dis 2016;16:161-8.

5. Lewis K. Challenges of antibiotic discovery. Microbe 2015;10:363-9.

6. Messacar K, Parker SK, Todd JK, et al. Implementation of rapid molecular infectious disease diagnostics: the role of diagnostic and antimicrobial stewardship. J Clin Microbiol 2017; 55:715-23.

7. Dyar OJ, Huttner B, Schouten J, et al. What is antimicrobial stewardship? Clin Microbiol Infect 2017;11:793-8.

8. Cox JA, Vlieghe E, Mendelson M, et al. Antibiotic stewardship in low-and middle-income countries: 'same, but different'? Clin Microbiol Infect 2017; 11:812-8. 